\title{
Indications, macroscopic and microscopic findings of out- patient hysteroscopy in a university hospital in Oman
}

\author{
Maryam Al-Shukri*, Naama Albadowi, Azza AlHarassi, Asha Nair, Bushra Ahmed, Wadha Al-Ghafri and V Gowri \\ Department of Obstetrics and Gynecology, Sultan Qaboos University Hospital, Oman
}

\begin{abstract}
Background: Out-patient hysteroscopy is an important tool of evaluation to women with reproductive difficulties like subfertility and recurrent pregnancy loss. It is also valuable to evaluate women with abnormal uterine bleeding. We conducted this retrospective study to evaluate the indications, macroscopic and microscopic findings of out-patients hysteroscopy at Sultan Qaboos University in Oman. The included patients were referred for outpatient hysteroscopy in the department of Obstetrics and Gynecology between February 2016 and December 2017. The electronic patient records were reviewed from the indications, findings on hysteroscopy and the results of endometrial biopsy if obtained. Descriptive analysis was performed.

Results: A total of 141 patients underwent out-patient hysteroscopy during the study period of 23 months. The age of the patients ranged from 21 to 58 years with a mean of $34 \pm 6.3$ years. The most frequent indication was recurrent pregnancy loss (RPL) in 33.3\% (47/141). The most common abnormality found on hysteroscopy was congenital anomalies of the uterine cavity in 27 patients (19.1\%).

When specifically looking at the 63(44\%) of women who had normal 2D-Trans-vaginal ultrasound scan (2D-TVS), 61.9\% of them had abnormal hysteroscopic findings including congenital anomalies in $13(20.6 \%)$. For congenital anomalies, 2D-TVS has a poor ability to pick-up those abnormalities with sensitivity of only $22 \%$. On biopsy, histological evidence of endometritis was found in $12.7 \%$ of patients whereas the bacterial endometrial culture was positive in (28/60) $46 \%$ of the samples.

Conclusion: In view of poor sensitivity of 2D-TVS for congenital anomalies and uterine synechiae; it is important to supplement pelvic ultrasound examination with hysteroscopic evaluation in symptomatic women. Our histological and microbiological evidence of endometritis is consistent with the fact that the offending organisms are the once usually contaminate the urogenital tract.
\end{abstract}

Abbreviations: 2D: 2 Dimentional; TVS: Trans Vaginal Ultrasound; RPL: Recurrent Pregnancy Loss; AUB: Abnormal Uterine Bleeding; IUD: Intra-Uterine Device.

\section{Background}

Following history and clinical examination, evaluating a woman with a gynecologic complaint classically involves ultrasound and hysteroscopy with endometrial biopsy when indicated [1]. Over the last thirty years; out-patient hysteroscopy -also known as office hysteroscopy- has been moving up the ladder in the hierarchy of the assessment and treatment of women with abnormal uterine bleeding, infertility, recurrent pregnancy loss and post-menopausal bleeding $[1,2]$. It is also gaining popularity among gynecologists for evaluation of painful periods due to adenomyosis and for assessment of the endometrial cavity when incidental findings are detected on imaging [3]. It was welcomed in the clinical practice and disseminated rapidly as the patients and their treating physician appreciated its efficient, reliable, safe and convenient "see and treat" approach $[1,2,4]$. The expert groups and the practice-guidelines moved from endorsing it as acceptable to being important to being a standard of care [5-8]. In a practice changing step, recently it being recommended as the first-line step in evaluating women with abnormal uterine bleeding (AUB) $[9,10]$.

Sultan Qaboos University hospital has been the second hospital in the country to introduce the service of out-patients hysteroscopy in Oman. This study is carried out to evaluate the indications and the findings of out-patients hysteroscopy in order to guide future practice and health services.

\section{Methods}

This is a retrospective cohort study of patients who were referred for evaluation by outpatient hysteroscopy in the department of Obstetrics and Gynecology at Sultan Qaboos University hospital, Oman, between February 2016 and December 2017 which was when the service was first made available. The ethical approval for the study was obtained from the ethics committee of the college of medicine and health science at sultan Qaboos university.

Patients' records were reviewed from the hospital information system. Data collected includes the patients' clinical history, indications of the out-patient hysteroscopy, their radiological evaluation by ultrasound, the procedure findings, and histopathology findings of the endometrial biopsy if it was performed. Statistical analysis was performed through Statistical Package for Social Sciences statistics 23.

${ }^{\star}$ Correspondence to: Maryam Nasser Al-Shukri, Senior consultant, Department of Obstetrics and Gynecology, Sultan Qaboos University Hospital, P.O. Box: 35, Postal Code:123, Muscat, Oman, E-mail: mnalshukri@gmail.com

Key words: hysteroscopy, uterine anomalies, endometritis, infertility, ultrasound

Received: December 09, 2019; Accepted: December 16, 2019; Published: December 20, 2019 


\section{Pre-procedure preparation}

All patients were first seen in the gynecology clinic where they were evaluated clinically by history, clinical examination and pelvic ultrasound.

\section{Pelvic ultrasound}

The findings of the most recent pelvic ultrasound prior to the hysteroscopy were recorded. The uterine cavity and endometrium were considered normal if ultrasound showed homogenous endometrial lining and distinct margins of the myometrium. The thickness of the endometrial lining is variable depending on the physiological changes accompanying the proliferative or the secretary phase of the menstrual cycle. Ultrasound was considered abnormal if it showed absence of central hyperechoic line, deformations of the endometrial lining, appearance of any structure with or without defined margins or variable echogenicity.

The Ultrasound was performed either in the radiology department or by the gynecologist during the out-patient visit. The details of endometrial thickness, the radiological echogenicity and the contents of endometrial cavity (polyps, fibroids, fluid) were reported in detail. Ultrasound Doppler studies were used to enhance the diagnostic accuracy of the ultrasound. In addition to endometrium and the cavity, ultrasound also evaluated the uterine wall, both ovaries and the pelvic cavity.

When the ultrasound showed an obvious intra-cavitary lesion requiring a hysteroscopic intervention, patients were counseled for operative hysteroscopy as in-patient. Out-patient hysteroscopy was offered primarily for diagnostic purposes. The procedure is explained to the patient in details and a written informed consent was obtained. Vaginal swab culture for bacteriology, Chlamydia and Gonorrhea were obtained and results are checked before the procedure in order to avoid the risk of post-procedure endometritis especially in patients with reproductive issues. If infection was detected, the patient was prescribed the appropriate antibiotic treatment. The procedure is usually scheduled in the proliferative phase of the menstrual cycle where the visualization of the endometrial cavity is optimal and prior to ovulation. If that was not possible due to timing constrains; the patient was instructed to avoid sexual intercourse after menses till the time of the procedure so an early pregnancy would not be a concern. On the day of the procedure; patients attend the day care unit without fasting.

\section{Procedure technique}

For out-patient hysteroscopy the patient is placed in lithotomy position. Hysteroscopy procedure started by introducing a hysteroscope by vaginoscopy technique through the cervical canal into the uterus into the uterine cavity. Normal saline was used as distention medium with free flow on an intravenous stand at a height above the patient's bed level and running free by gravity. No anesthetic or sedative were used.

Table 1. Number of patients and the type of evaluation performed

\begin{tabular}{|c|c|c|c|c|c|c|c|c|}
\hline & \multicolumn{2}{|c|}{ Endometrial biopsy } & \multirow{2}{*}{ Histopathology } & \multirow{2}{*}{ Normal histology } & \multirow{2}{*}{ Inflammation } & \multirow{2}{*}{ Bacterial culture } & \multirow{2}{*}{ No growth } & \multirow{2}{*}{ Positive growth } \\
\hline & No. & $\%$ & & & & & & \\
\hline Infertility & $26 / 42$ & 61.9 & 24 & 20 & 4 & 18 & 9 & 9 \\
\hline RPL* & $39 / 47$ & 83.0 & 37 & 31 & 4 & 37 & 20 & 17 \\
\hline AUB** & $32 / 46$ & 69.5 & 29 & 24 & 3 & 8 & 6 & 2 \\
\hline Total & & 97 & 90 & 75 & 11 & 63 & 35 & 28 \\
\hline
\end{tabular}

*Recurrent Pregnancy Loss

**Abnormal Uterine Bleeding them was 2 millimeter $(\mathrm{mm})$ in diameter in an operative sheet of 3.6 $\mathrm{mm}$ outer diameter. The other was $2.9 \mathrm{~mm}$ diameter with an operative sheet of $4 \mathrm{~mm}$ outer diameter. Vaginoscopy approach was used. In this approach there is no extra instruments used to visualize the cervix like a vaginal speculum or cervical tenaculum. Any pain experienced by the patient was most likely to be during passage of the scope through a narrowed internal cervical ostium. Visual observation of the uterine cavity is performed with simultaneous explanation of the procedure and findings to the patient while live images displayed on a monitor for both the patient and the gynecologist. A hysteroscopy directed biopsy (under hysteroscopic guidance) is obtained if a lesion was visualized for histopathologic evaluation of bacterial culture when indicated. Patients were discharged home at the same day immediately after the procedure. If significant pain was expressed by the patient during the procedure, the procedure was immediately terminated.

\section{Results}

Of the 165 bookings for hysteroscopy, 34 (23\%) either did not show up for the procedure or were cancelled and some were rescheduled for another date. Some of the reasons for cancellations were menstruation, evidence of an ongoing vaginal infection, suspicion of pregnancy and non-compliance with advised abstinence if indicated.

A total of 141 patients underwent out-patient hysteroscopy and further endometrial evaluation during the study period as in Table 1. The age of the patients ranged from 21 to 58 years with a mean age of $34 \pm 6.3$ years. The most frequent indications were recurrent pregnancy loss in $33.3 \%(47 / 141)$ abnormal uterine bleeding (AUB) in $32.2 \%$ $(46 / 141)$ and infertility in $29.8 \%(42 / 141)$. Other indications were less frequent like incidental findings of thick endometrium in a postmenopausal woman in 2, postmenopausal bleeding in 2, removal of intra-uterine device (IUD) in 2 patients and second look hysteroscopy following a resection of uterine septum in 1 patient and assessment of the extent of type of submucous fibroid in 1 patient.

In $15(10.6 \%)$ women the procedure was not completed due to significant pain on attempting navigation through the cervical canal. Of those women where the procedure not completed; 6 women were with infertility, 3 with recurrent pregnancy loss and 3 with abnormal uterine bleeding. The other 2 women were post-menopausal, one with bleeding and another with incidental finding of a thick endometrium.

Seventy-five women (53.2\%) had abnormal hysteroscopic findings. The most common abnormality found on hysteroscopy was congenital anomalies of the uterine cavity. Those anomalies were found in 27 patients (19.1\%); namely bicornuate uterus in 3, uterine septum in 15 , arcuate uterus in two women, narrow cavity and classical T-Shaped in one woman each. Twenty-five (17.7\%) women had endometrial polyps, and $18(12.8 \%)$ had fibroids affecting the cavity.

To assess the benefit of out-patient hysteroscopy in patients with normal 2D ultrasound, we specifically looked at those women who had 
an abnormal hysteroscopic evaluation and a normal finding on Transvaginal 2-D ultrasound. There were $63(44.7 \%)$ in this group. Thirtynine $(61.9 \%)$ of the women who had abnormal hysteroscopic findings had more than one hysteroscopic findings and normal 2D ultrasound. Findings included congenital anomalies in 13 (20.6\%), endometrial synechiae in $7(11.1 \%)$, small endometrial polyps in $8(12.7 \%)$, and fibroids affecting the cavity in 5 (7.9\%) women.

Overall, in symptomatic patients, the sensitivity of ultrasound to find an abnormality was $54 \%$ with specificity of $52 \%$. However, for congenital anomalies, 2D ultrasound had a poor ability to pick-up those abnormalities with sensitivity of only $22 \%$.

\section{Endometrial biopsy}

Endometrial biopsy was performed for women with abnormal uterine bleeding or reproductive difficulty to detect histological abnormalities or investigate the presence of endometritis by histological evidence and or positive bacterial culture respectively. Two thirds $(100 / 141)$ of women had endometrial biopsy. Of the 41 who did not have a biopsy; $23(56 \%)$ had an anatomical abnormality that might explain their symptom ( 4 had polyps, 11 had uterine anomaly and 4 had fibroids). The other women had cervical stenosis and were scheduled for hysteroscopy as inpatients. Endometrial samples in two women were insufficient for assessment.

Of the 46 women with abnormal uterine bleeding; 8 did not have an endometrial biopsy but had anatomical abnormalities that required another intervention. Of the 32 Women who had endometrial biopsy, three women had evidence of endometritis, one had complex endometrial hyperplasia, one had insufficient sample for evaluation and the rest of them had normal histological studies

For the 89 women with reproductive difficulties (infertility and recurrent pregnancy loss); 71 (80.0\%) had endometrial biopsy. Of those who had endometrial biopsy, 9/71 (12.7\%) had histological evidence of endometritis, one had simple endometrial hyperplasia and one had insufficient sample for assessment.

The organisms identified on all the bacterial cultures of the endometrium is depicted in Table 2. The eight women who had histological evidence of endometritis, 3 had no growth on the bacterial culture, 5 had positive bacterial growth, (2 grew staphylococcus hemolyticus, 1 staphylococcus epidermides, 1 enterococcus faecalis,

Table 2. The type of organisms isolated in endometrial culture

\begin{tabular}{|c|c|}
\hline Organisms detected in the biopsies & No. of isolates \\
\hline staphylococcus epidermidis & 5 \\
\hline staphylococcus aureus & 1 \\
\hline staphylococcus agalactiae & 1 \\
\hline staphylococcous hemolyticus & 5 \\
\hline staphylococcus hominis & 2 \\
\hline klebsiela pneumonia & 2 \\
\hline Escherichia coli & 1 \\
\hline enterococcous faecalis & 5 \\
\hline enterobacter aerogenes & 1 \\
\hline corynebacterium amyocolatum & 1 \\
\hline bacilus circulans & 1 \\
\hline bacilus cereus & 1 \\
\hline acinetobactecter baumannii & 1 \\
\hline Group B streptococcus & 1 \\
\hline streptoccous viridans & 1 \\
\hline streptococcus pyogenes & 1 \\
\hline Total & 27 \\
\hline
\end{tabular}

1 bacillus cereus). We also attempted to correlate between positive bacterial culture and the findings of endometritis on histopathology. The degree of agreement was insignificant.

\section{Discussion}

\section{Optimizing utilization of out-patient services}

The introduction of out-patient hysteroscopy to our hospital was a positive step to expedite diagnosis and management of those patients who needed work-up for the reproductive difficulties or abnormal uterine bleeding [11]. Of the scheduled patients, we had $23 \%$ cancelation rate. Unfortunately, we were not able to find comparison on reported cancelation rate for out-patient hysteroscopy. This figure should encourage us to improve the adopted education and communication strategy with the patients [12]. It should also aid in brainstorming for other options like a "one stop clinic" $[1,13]$.

\section{Findings on macroscopic evaluation of the uterine cavity}

For the procedure and as a referral hospital, our top three indications for outpatient hysteroscopy are recurrent pregnancy loss, AUB and infertility were $33 \%, 31 \%$ and $29 \%$ respectively. This somehow differs from other centers that published their work. Example is Tangri et al showing infertility as the most common indication (49\%.0\%), followed by abnormal uterine bleeding (36.6\%) and postmenopausal bleeding $(8.3 \%)$. The discrepancy in the proportions can be explained by the nature of the local practice [14]. In our hospital there is a special referral clinic for recurrent pregnancy loss whereas in the compared center in Tangri et al; there was a fertility center that uses out-patient hysteroscopy as routine part of their evaluation for all patients with infertility $[14,15]$. In our study the proportion of post-menopausal women was much smaller as that reflects the general population of the country where women older than 50 years of age are about $2 \%$ of the total population in Oman in 2017 [16].

In $10.6 \%$ of our patients; cervical navigation resulted in significant pain consequently the procedure was aborted. This number is multifactorial. Patient related factors like patient age, anxiety level, pain threshold, previous reproductive history and the use of analgesia. Instruments related factors including the type and diameter of the scope. Physician related factors like the experience and threshold to empathize with the patients pain to stop the navigation. It is known that the procedure related pain and failure is less with mini-hysteroscopes (diameter of $3.5 \mathrm{~mm}$ or less) compared to those of $5 \mathrm{~mm}$ in diameter [17]. Nulliparity, presence of cervical pathology and duration of the procedure longer than 2 minutes were found to be significantly related to severe and intolerable pain $[11,18,19]$. In the same study, $17.3 \%$ of patients reported severe pain but the procedure was completed and $2.3 \%$ reported intolerable pain where the procedure was terminated [19].

In our study; $53.2 \%$ of women had abnormality on hysteroscopy. The proportion of women with an abnormal finding on hysteroscopy depends on the selected study population but mostly reported between $38-78 \%[2,14,20]$. The Reported to be the most common abnormality found on hysteroscopy was congenital uterine anomalies in $19 \%$ of the patients. This number makes sense since our study population are high risk population for uterine anomalies including women with recurrent pregnancy loss and infertility [13].

\section{Comparing normal ultrasound to hysteroscopy findings}

When comparing ultrasound and hysteroscopic findings, $44.7 \%$ of the study population had a normal $2 \mathrm{D}$ ultrasound and an abnormality 
on hysteroscopy. The findings were mostly congenital abnormality and intra-uterine adhesions for which ultrasound is known to have a poor sensitivity and specificity. Overall, in our study of symptomatic patients, the ultrasound sensitivity to find an abnormality was $54 \%$ with specificity of $52 \%$. However, for congenital anomalies, 2-D ultrasound has a poor ability to pick-up those abnormalities with sensitivity of only $22 \%$.

Ultrasound reported to have a sensitivity of $85-89 \%$ and a specificity of 56\%-91.8\% [15,21]. In another study; there was a difference between an initial 2DTVS and a 2D-TVS by an expert. general detection of uterine abnormalities, compared with initial 2D-TVS (77.8\%), expert 2D-TVS (90.6\%), 2D-SIS (94.0\%), and 3D-TVS (97.4\%) [22]. Our numbers are lower compared to the reported studies. The explanation for that are the ultrasound done in our clinic are done by doctors with different levels of training in ultrasound. Although most of the ultrasounds performed were TVS but not all were, and that explains why likely our numbers are lower compared to the reported once.

\section{Microscopic evaluation of the endometrium}

When performing endometrial biopsy in our patients, it has been the routine that the biopsy is taken after the hysteroscopy. Although there are studies that looked at what is the optimal order of hysteroscopy and the endometrial biopsy and the difference was not significant [23]. We believe hysteroscopy may affect the biopsy results by 2 contradicting mechanisms: hysteroscopy may further wash out endometrial bacteria and decrease its load. The other mechanism is hysteroscopy might also push in vaginal contaminants into the endometrial cavity.

Women with reproductive difficulty had biopsy for culture and histopathologic examination. Those with AUB had biopsy for histopathologic examination. Hysteroscopy and endometrial biopsy are considered gold standard in evaluating women with AUB $[1,4]$. The results reflect that we were keener to perform endometrial biopsy unless there was an abnormality that might explain the patient symptoms.

A histologic evidence of endometritis was detected in $12.7 \%$ of women with reproductive difficulty. There has been an ongoing interest in detection of endometritis in women with reproductive difficulties especially recurrent pregnancy loss [24,25]. Chronic endometritis does not usually present with "inflammatory symptoms of pain, fever or uterine tenderness" [26,27]. The causative organisms are mostly bacterial that usually ascends from urogenital or gastrointestinal tracts [25]. It is reported to be $10-14 \%$ when diagnosed histologically based on the presence of plasma cells $[24,25]$. Our results were representative of such a range. In another study, the group specific prevalence was found to be $10.8 \%$ in recurrent miscarriage, $7.7 \%$ in recurrent implantation failure and $10.4 \%$ in infertility [28]. The offending organisms we identified were same as identified in other studies [24].

This study emphasizes the increasing value of out-patient hysteroscopy in terms of macroscopic and microscopic evaluation of uterine cavity. It also addresses the full path of patient evaluation clinical, radiologic, hysteroscopic, histopathologic and microbiologic features that enforces the high-level individualized decision required for further management plan. However; the fact that it is a retrospective study comes as a short coming. Another short coming of this study is that in some categories the numbers were small that did not allow sufficient statistical evaluation.

\section{Conclusion}

This review of our patients confirmed the need to provide outpatient hysteroscopy to high risk population to expedite diagnosis and management especially where $2 \mathrm{D}$ ultrasound has shortcomings in detecting uterine anomalies. The fact that some authorities in the field of gynecology puts hysteroscopy as a primary investigation in evaluating women with abnormal uterine bleeding further drives the need for out-patient hysteroscopy services.

\section{Declarations}

\section{Competing interests}

All authors declare that they have no competing interests

\section{Funding}

None

\section{Authors' contributions}

- Maryam Al Shukri: conception of the idea, design of the study, data analysis and drafted the manuscript.

- Naama Al Harrasi \& Azza Al Badwawi: data collection and analysis.

- Asha Nair\& Bushra Ahmed: Data collection, critical revision of manuscript.

- Wadha Al Ghafri\& V Gowri: data interpretation and critical revision of manuscript.

\section{References}

1. Darwish AM, Sayed EH, Mohammad SA, Mohammad II, Hassan HI (2012) Reliability of out-patient hysteroscopy in one-stop clinic for abnormal uterine bleeding. Gynecol Surg 9: 289-295.

2. Yen CF, Chou HH, Wu HM, Lee CL, Chang TC (2019) Effectiveness and appropriateness in the application of office hysteroscopy. J Formos Med Assoc 1-8.

3. Revel A, Shushan A (2002) Investigation of the infertile couple: Hysteroscopy with endometrial biopsy is the gold standard investigation for abnormal uterine bleeding. Hum Reprod 17: 1947-1949. [Crossref]

4. Moawad NS, Santamaria E, Johnson M, Shuster J (2014) Cost-effectiveness of office hysteroscopy for abnormal uterine bleeding. JSLS 18: e2014. [Crossref]

5. Rowe P, Hargreave T, Mellows H (1993) WHO manual for the standardized investigation and diagnosis of the infertile couple. Press Synd Univ Cambridge.

6. American College of Obstetricians and Gynecologists (2011) Technology assessment No. 7: Hysteroscopy. Obstet Gynecol 117: 1486-1491.

7. Italian Society of Gynecological Endoscopy (linee guida per l'isteroscopia office) (2013) Practical guideline in office hysteroscopy.

8. Deffieux X, Gauthier T, Menager N, Legendre G, Agostini A, et al. (2014) Hysteroscopy: Guidelines for clinical practice from the French College of Gynaecologists and Obstetricians. Eur J Obstet Gynecol Reprod Biol 178: 114-122. [Crossref]

9. National Institute for Health and Care Excellence (NICE). Heavy Menstrual Bleeding: assessment and management [Internet]. NICE NG88. 2018. Available from: www.nice. org.uk/guidance/ng88

10. Ramshaw N, Narayansingh G (2019) The implications of hysteroscopy in the updated guidelines on heavy menstrual bleeding from the UK National Institute for Health and Care Excellence (NICE). Case Rep Women's Health 22: e00117. [Crossref]

11. Bennett A, Lepage C, Thavorn K, Fergusson D, Murnaghan O, et al. (2019) Effectiveness of outpatient versus operating room hysteroscopy for the diagnosis and treatment of uterine conditions: A systematic review and meta-analysis. $J$ Obstet Gynaecol Canada 41: 930-941. [Crossref]

12. Barlow DH (2019) Optimizing office hysteroscopy. Fertil Steril 111: 465-466. [Crossref]

13. Koskas M, Mergui JL, Yazbeck C, Uzan S, Nizard J (2010) Office hysteroscopy for infertility: A series of 557 consecutive cases. Obstet Gynecol Int 2010: 168096. [Crossref] 
14. Tangri MK, Lele P, Kapur K, Kapur A, Chhabra N, et al. (2017) Role of office hysteroscopy in gynecology: retrospective observational study at a tertiary care hospital. Int J Reprod Contraception Obstet Gynecol 6:111-116.

15. El Huseiny AM, Soliman BS (2013) Hysteroscopic findings in infertile women: A retrospective study. Middle East Fertil Soc J 18: 154-158.

16. tradingeconomics. Oman Population | 1960-2017 | Data | Chart | Calendar | Forecast | News [Internet]. Tradingeconomics.Com. 2017. Available from: https:// tradingeconomics.com/oman/population

17. Cicinelli E (2010) Hysteroscopy without anesthesia: Review of recent literature. $J$ Minim Invasive Gynecol 17: 703-708.

18. Kopitović V, Budakov D, Pjević A, Pop-Trajković S, Djurdjević S, et al. (2012) Results and experiences after 2000 performed hysteroscopies. Srp Arh Celok Lek 140(11-12): 728-731. [Crossref]

19. Zayed SM, Elsetohy KA, Zayed M, Fouda UM (2015) Factors affecting pain experienced during office hysteroscopy. Middle East Fertil Soc J 20: 154-158.

20. Yang JH, Chen MJ, Yang PK (2019) Factors increasing the detection rate of intrauterine lesions on hysteroscopy in infertile women with sonographically normal uterine cavities. J Formos Med Assoc 118(1P3): 488-493. [Crossref]

21. Parry JP, Isaacson KB (2019) Hysteroscopy and why macroscopic uterine factors matter for fertility. Fertil Steril 112: 203-210. [Crossref]
22. Ludwin A, Pityński K, Ludwin I, Banas T, Knafel A (2013) Two- and Two- and three-dimensional ultrasonography and sonohysterography versus hysteroscopy with laparoscopy in the differential diagnosis of septate, bicornuate, and arcuate uteri. $J$ Minim Invasive Gynecol 20: 90-99. [Crossref]

23. Sarkar P, Mikhail E, Schickler R, Plosker S, Imudia AN (2018) optimal order of successive office hysteroscopy and endometrial biopsy for the evaluation of abnormal uterine bleeding: a randomized controlled trial. Obstet Gynecol Surv 73: 207-208. [Crossref]

24. Cicinelli E, Matteo M, Tinelli R, Pinto V, Marinaccio M, et al. (2019) Chronic endometritis due to common bacteria is prevalent in women with recurrent miscarriage as confirmed by improved pregnancy outcome after antibiotic treatment.

25. Sahasrabudhe N, Mobasseri M, Reznik SE, Williams Z (2017) Chronic endometritis and recurrent pregnancy loss. Curr Obstet Gynecol Rep 6: 55-61.

26. Di Spiezio Sardo A, Palma F, Calagna G, Zizolfi B, Bifulco G (2016) Chronic endometritis. In: Genital infections and infertility. InTech 48-52.

27. Kitaya K, Takeuchi T, Mizuta S, Matsubayashi H, Ishikawa T (2018) Endometritis: new time, new concepts. Fertil Steril 110: 344-350. [Crossref]

28. Liu Y, Chen X, Huang J, Wang CC, Yu MY, et al. (2018) Comparison of the prevalence of chronic endometritis as determined by means of different diagnostic methods in women with and without reproductive failure. Fertil Steril 109: 832-839. [Crossref]

Copyright: $@ 2019$ Al-Shukri M. This is an open-access article distributed under the terms of the Creative Commons Attribution License, which permits unrestricted use, distribution, and reproduction in any medium, provided the original author and source are credited. 